\section{Vinflunin in die Leitlinien der EAU aufgenommen}

Die European Association of Urology (EAU) hat Vinflunin $\left(\right.$ Javlor $^{\circledR}$ ) in ihre Leitlinien zur Therapie des metastasierten Urothelkarzinoms aufgenommen (Guidelines on Bladder Cancer 2010; www.uroweb.org): Vinflunin wird als Standard in der Zweitlinientherapie bei Patienten mit Progression nach einer platinhaltigen Behandlung empfohlen. In einer randomisierten Phase-III-Studie konnte bei Patienten mit Versagen einer platinhaltigen Erstlinientherapie durch die Therapie mit Vinflunin im Vergleich zu Best Supportive Care eine signifikante Verlängerung des medianen Gesamtüberlebens gezeigt werden (6,9 Monate vs. 4,3 Monate; $p=0,0403$; Bellmunt J et al., 2009, J Clin Oncol 27: 4454-4461). Quelle: Informationen der Pierre Fabre GmbH

\section{MSD-Stipendium 2009 Onkologie}

Für sein Forschungsprojekt „In vitro and in vivo characterization of brain tumor stem-like cell (BTSC) population from high grade gliomas derived from neurogenic and non-neurogenic regions of the human central nervous system" erhielt Jaroslaw Maciaczyk, Freiburg, das MSD-Stipendium 2009 Onkologie in Höhe von 12.000 Euro. Quelle: Informationen der MSD SHARP \& DOHME GMBH

\section{Schilddrüsenkarzinom:}

\section{Ablation von} \section{Schilddrüsenrestgewebe}

\section{Die Zulassung für Thyrogen ${ }^{\circledast}$ (rekom-} binantes humanes Thyreoidea-stimulierendes Hormon, Thyrotropin alfa) bei der ablativen Radioiodtherapie von Schilddrüsenkarzinomen wurde erweitert. Der Einsatz des Präparats ist in Kombination mit lod 131 zur Ablation von Schilddrüsenrestgewebe bei allen Patienten mit differenziertem Schilddrüsenkarzinom ohne bekannte Fernmetastasen möglich. Bislang war die Anwendung des Präparats auf Niedrigrisiko-Patienten beschränkt.

Quelle: Informationen der Genzyme GmbH

Best Practice Award 2010

\title{
Mehr Dialog in der Onkologie
}

Mit dem Best Practice Award „Mehr Dialog bei Krebs" werden Projekte gefördert, die die Kommunikation zwischen Ärzten, Pflegekräften und Patienten mit Tumorerkrankungen vorbildlich unterstützen. Der Best Practice Award wurde von Novartis Oncology in Kooperation mit der Deutschen Krebsgesellschaft e.V. im Rahmen des 29. Deutschen Krebskongresses in Berlin zum dritten Mal verliehen.

Den 1. Preis des Best Practice Awards 2010, dotiert mit 5.000 Euro, erhielt die Selbsthilfegruppe Berg und Tal e.V., vertreten durch Klaus F. Röttger, Bochum - Essen. Die Selbsthilfegruppe für Patienten und Angehörige vor und nach Knochenmark- oder Stammzelltransplantation verfolgt das Ziel, die psychosoziale Betreuung im Rahmen der Akutbehandlung und der Nachsorge zu fördern, die Prävention und Rehabilitation zu verbessern und die Zusammenarbeit von Betroffenen und Fachkräften zu fördern. Die Gruppe setzt sich für eine offene und vertrauensvolle Kommunikation zwischen Ärzten, Patienten und Angehörigen ein und bemüht sich um eine möglichst multimediale Aufbereitung vielfältiger Informationen und Lebenshilfen. Das vielfältige Angebot der Selbsthilfegruppe ist unter www.bergundtal-ev.de zu finden.

Der Verbund Pädiatrische Onkologie Weser-Ems, koordiniert durch Hermann Müller, Oldenburg, wurde mit dem 2. Preis ausgezeichnet. In diesem Verbund haben sich Pädiater verschiedener Kinderkliniken und Kinderabteilungen im Weser-Ems-Bezirk, Selbsthilfegruppen und ambulante Pflegedienste zusammengeschlossen, um die Betreuung pädiatrisch-onkologischer Patienten in diesem flächenmäßig großen Einzugsgebiet zu verbessern: Die Patienten und Angehörigen erhalten somit eine möglichst wohnortnahe, koordinierte und kompetente Betreuung.

Den 3. Preis erhielt die Anlaufstelle für Patienten mit Ösophaguskarzinom unter Leitung von Elfriede Bollschweiler, Köln. Das Ziel dieses Projekts ist insbesondere die Information von Patienten mit Ösophaguskarzinom über den aktuellen Wissensstand. Zu diesem Zweck wurden beispielsweise eine Internetplattform (www.speiseroehrenkrebs.uni-koeln.de) und eine Telefonhotline für Ratsuchende eingerichtet. $R F$ Quelle: Veranstaltung von Novartis Oncology

\section{Essenzielle Thrombozythämie}

\section{Bestmögliches Management}

Bei guter Therapie haben Patienten mit essenzieller Thrombozythämie (ET) eine fast normale Überlebenserwartung. Dennoch stellt die Erkrankung bei Diagnose und Therapie erhebliche Anforderungen an den Hämatologen, v.a. wenn er nur wenig Erfahrung mit diesen Patienten hat.

Neben den Symptomen und Komplikationen der ET können behandlungsbedingt offene, vielfach aber auch versteckte Nebenwirkungen, wie z.B. Hautveränderungen, kardiologische oder psychiatrische Begleiterscheinungen, auftreten. Diesem Problem hat sich eine von der Shire Pharmaceuticals Group initiierte interdiszip- linäre Arbeitsgruppe von zehn internationalen Experten gewidmet und relevante Informationen erarbeitet, die zusätzlich in einem größeren Kreis mit Ärzten aus ganz Europa abgestimmt wurden. Die Ergebnisse ermöglichen dem Hämatologen ein bestmögliches ET-Management und geben Anregungen für die Vermeidung bzw. Behandlung auftretender unerwünschter Ereignisse. Die erarbeiteten Inhalte werden dieses Jahr einen besonderen Schwerpunkt bei den von Shire initiierten hämatologischen Symposien einnehmen. Für weitere Informationen kontaktieren Sie bitte Shire Deutschland GmbH 030 / 206582-0. Quelle: Informationen der Shire Deutschland GmbH 